\title{
Associations of Sex Hormone-binding Globulin with Bone Mineral Density Among US Adults, NHANES 2013-2016
}

\author{
Fan Yang $\mathbb{D}^{1,2}$ \\ Dawei Yang ${ }^{3}$ \\ Yinan Zhou ${ }^{4}$ \\ Jianjun $\mathrm{Wu} \mathbb{D}^{1,2}$
}

'Department of Cardiology, The Second Affiliated Hospital of Harbin Medical University, Harbin, People's Republic of China; ${ }^{2}$ Key Laboratory of Myocardial Ischemia, Ministry of Education, Harbin Medical University, Harbin, People's Republic of China; ${ }^{3}$ Department of Orthopedics, The Fourth Affiliated Hospital of Harbin Medical University, Harbin, People's Republic of China; ${ }^{4}$ Department of Digestive Internal Medicine, The First Affiliated Hospital of Harbin Medical University, Harbin, People's Republic of China
Correspondence: Jianjun Wu

Department of Cardiology, The Second Affiliated Hospital of Harbin Medical University, Harbin, Province Heilongjiang, I5000I, People's Republic of China Tel +86-I36845I0900

Email drwujianjun@sina.com

Fan Yang

The Key Laboratory of Myocardial Ischemia, Chinese Ministry of Education, Harbin, Province Heilongjiang, I5000I,

People's Republic of China

Tel +86-18545850825

Email yangfana@sina.com
Background: Bone metabolism can be influenced by sex steroid hormones. However, the relationship between sex steroid hormones and bone mineral density (BMD) remains inconsistent. Our study explored existing evidence of the association between sex hormones, blood glucose and BMD.

Methods: We conducted a cross-sectional study of 6434 participants aged 18-80 years in the National Health and Nutrition Examination Survey (NHANES) 2013-2016 to assess the association between SHBG and BMD. The multivariable linear regression models were performed to evaluate the associations between testosterone, estrogen, sex hormonebinding globulin (SHBG) and BMD.

Results: On adjustment for traditional risk factors (age, BMI, ALB, ALK, ASP, calcium, cholesterol, potassium, sodium, total protein, uric acid), SHBG could be regarded as an independent predictor for BMD, while fasting blood glucose did not. SHBG levels were significant associated with BMD in the multivariate model; however, it will be a different effect between the genders. On comparative analysis, high level SHBG was predictive of lumbar BMD irrespective of other two sex steroid hormones.

Conclusion: This study indicated that SHBG may improve the predictive value for bone loss in adults.

Keywords: sex steroid hormones, SHBG, bone mineral density, NHANES

\section{Introduction}

Since the number of senior citizens increased dramatically especially in developed countries, the burden of life-threatening conditions is increased. Osteoporosis is a systemic skeleton disease characterized by an imbalance of bone metabolism and high risk of fractures, whose clearly fundamental pathophysiological is mechanical microenvironment. ${ }^{1,2}$ As an age-related disease, osteoporosis has multiple similar characteristics with diabetes, cardiovascular disease and fractures that affects an estimated 44 million Americans over the age of 50 years, leading to significant morbidity, mortality, and disability all over the world. ${ }^{3,4}$ However, the clear understanding of associated characteristics between osteoporosis and body metabolism has not been fully revealed.

Organ to organ crosstalk greatly impacts metabolism. The interrelation between cortisol and bone metabolism is complex. ${ }^{5,6}$ Sex hormone deficiency in both genders results in bone loss and bone strength or fracture risk depending on bone quality, as well as bone mineral density (BMD). ${ }^{7,8}$ BMD levels are influenced by environmental 
and genetic factors and closely associated with osteoporosis or bone fracture. The association between serum sex steroid hormones levels and BMD in adults has been investigated previously. And most studies have demonstrated that there is a negative relationship between sex hormone-binding globulin (SHBG) levels and BMD. ${ }^{9-11}$ Sex steroid hormones are responsible for the normal working of heart and kidney, and are produced by ovaries in females and testes in males. $^{12}$ Sex hormone-binding globulin (SHBG) has been regarded as a specific plasma transport glycoprotein of sex steroid hormones, including testosterone, estrogen, and progesterone. ${ }^{13}$ Any association between SHBG and BMD remain inconclusive. In a cross-sectional study of nondiabetic men, SHBG levels were inversely associated with glycated hemoglobin level, suggesting a relationship between SHBG and glucose homeostasis among individuals without diabetes. ${ }^{14}$ In addition to epidemiologic studies demonstrating a consistent relationship between decreased levels of serum SHBG and incident T2DM, recent genetic studies also reveal that transmission of specific polymorphisms in the SHBG gene influence risk of T2DM. Several polymorphisms in the human SHBG gene has been found that genotype analysis of the exon 2 SHBG SNPs, rs6257 and rs6259, are associated with insulin resistance and reducing bone mineral density. ${ }^{15}$ A widely accepted theory about how SHBG is involved in bone metabolism is based on its anti-estrogenic effect. Higher SHBG binds to estrogen to reduce its biologically active form, which consequently reduces $\mathrm{BMD}$ and increases fracture risk. Because of the limitation of retrospective studies, and the changes in SHBG and BMDs over time were not examined, therefore, whether their changes are involved in the interactions is still controversial. At present, the most important known function of SHBG appears to be to regulate the bioavailability of circulating hormones. Therefore, we add to existing evidence of the association between sex hormones, blood glucose and BMD, using population data from NHANES 2013-2016.

\section{Methods}

\section{Data Sources}

Data was extracted from the 2013-2016 National Health and Nutrition Examination Survey (NHANES, n=6343), which was designed and conducted by the National Center for Health Statistics (NCHS) and freely available on the Internet for researchers throughout the world. For baseline statistical analysis, participants $(n=6343)$ were grouped into: young $(n=2137)$, mid-age $(n=3033)$, and elderly $(n=1264)$. For comparing sex steroid hormone (nmol/L) with fasting glucose to BMD, participants were divided into four groups. Group $1(n=100)$ : FBG $\leq 6.0$ and low sex steroid hormone level; group $2(n=46)$ : FBG $>6.0$ and low sex steroid hormone level; group $3(n=169)$ : FBG $\leq 6.0$ and high sex steroid hormone level; group $4(n=43)$ : FBG $>6.0$ and high sex steroid hormone level. For exploring differences between genders, participants were divided into males $(n=3175)$ and females $(n=3259)$. The survey protocol was approved by the Institutional Review Board of the Centers for Disease Control and Prevention (protocol \#9812 and protocol \#2005-2006). The National Center for Health Statistics ethics review board approved the protocols (https://www.cdc.gov/nchs/nhanes/irba98.htm). Written informed consent was obtained from all participants.

\section{Data Collection and Definition}

All participants have provided information on demographics (age, sex, and PIR), laboratory data (fasting serum glucose, mmol/L; blood sex hormone-binding globulin, SHBG, nmol/L), physical examination data (standing height, $\mathrm{cm}$; weight, $\mathrm{kg}$; body mass index, BMI, $\mathrm{kg} / \mathrm{m}^{2}$; systolic blood pressure, $\mathrm{SBP}, \mathrm{mmHg}$; diastolic blood pressure, DBP, mmHg; bone mineral density, BMD, g/ $\mathrm{cm}^{2}$; albumin, g/dL; alkaline phosphatase, IU/L; aspartate aminotransferase, IU/L; alanine aminotransferase, IU/L; bicarbonate, $\mathrm{mmol} / \mathrm{L}$; total calcium, $\mathrm{mg} / \mathrm{dL}$; cholesterol, $\mathrm{mg} / \mathrm{dL}$; globulin, g/dL; potassium, mmol/L; sodium, mmol/L; phosphorus, $\mathrm{mg} / \mathrm{dL}$; total bilirubin, $\mathrm{mg} / \mathrm{dL}$; total protein, $\mathrm{g} / \mathrm{L}$; uric acid, umol/L; total testosterone, $\mathrm{ng} / \mathrm{dL}$; estradiol, $\mathrm{pg} / \mathrm{mL}$ ) are all described on the NHANES website at https://wwwn.cdc.gov/Nchs/Nhanes/continuousnhanes/ default.aspx?BeginYear=2013.Serum glucose (nonfasting) was measured by means of a Roche/Hitachi Chemistry Analyzer (Roche Diagnostics, Indianapolis, IN, USA). BMI was defined as mass $(\mathrm{kg})$ divided by the square of height $\left(\mathrm{m}^{2}\right)$.

\section{Measurement of SHBG}

The laboratory method for detecting serum SHBG in NHANES was described in detail on website (https:// wwwn.cdc.gov/Nchs/Nhanes/2013-2014/TST_H.htm).

SHBG in blood affects the amount of biologically active steroid hormones, and its concentrations were determined using Roche/Hitachi cobase 411 analyzer from the serum sample and not taken from patients receiving therapy with 
high biotin doses (i.e. $>5 \mathrm{mg} /$ day) until at least eight hours following the last biotin administration (https://wwwn.cdc. gov/nchs/data/nhanes/2013-2014/labmethods/TST H MET_Sex_Hormone_Binding_Globulin.pdf).

\section{Measurement of Dual-energy X-ray Absorptiometry - Whole Body}

The whole body scans were acquired on the Hologic Discovery model A densitometers (Hologic, Inc., Bedford, MA, USA), using software version Apex 3.2. The radiation exposure from DXA whole body scans is extremely low at less than $20 \mathrm{uSv}$. The Hologic software, APEX v 4.0 (Hologic) was used to analyze whole body scans acquired in 2013-2016. Further details of the DXA examination protocol are documented in the Body Composition Procedures Manual located on the NHANES website.

\section{Statistical Analysis}

All continuous variables were presented as mean \pm standard deviation. An initial descriptive analysis utilized chi-squared and ANOVA tests to examine significant differences in baseline demographic and disease characteristics across levels of fasting blood glucose and diabetes status. The one-way ANOVA, Kruskal-Wallis H-test and chi-squared tests were used to determine any statistical differences between subgroups. Statistical analyses were performed using SPSS Version 21 (IBM Corporation, Armonk, NY, USA) and Stata V12.0 (Stata Corporation, College Station, TX, USA).

\section{Results}

\section{Associations of Age with Bone Mineral Density Related Index}

A total of 6434 subjects were included in the present study (Figure 1). We divided the participants into three groups according to age: groups of young, mid-age, and elderly were compared to our study (Table 1). On the whole, we observed a significant age-dependent changes in BMD, fasting glucose and testosterone. Albumin, ALA, blood calcium, cholesterol, serum sodium, phosphorus, and total bilirubin have the obvious difference with increasing age ( $p<0.05$ for each). In addition, we found an age-dependent decrease of testosterone and estradiol, which is consistent with previous studies. There seems to be obvious decrease in total BMD, thoracic spine $\mathrm{BMD}$, lumbar spine $\mathrm{BMD}$, or pelvis $\mathrm{BMD}$ between mid-age and elderly $(p<0.01)$.
Flow chart of sample selection from the NHANES 2013-2016

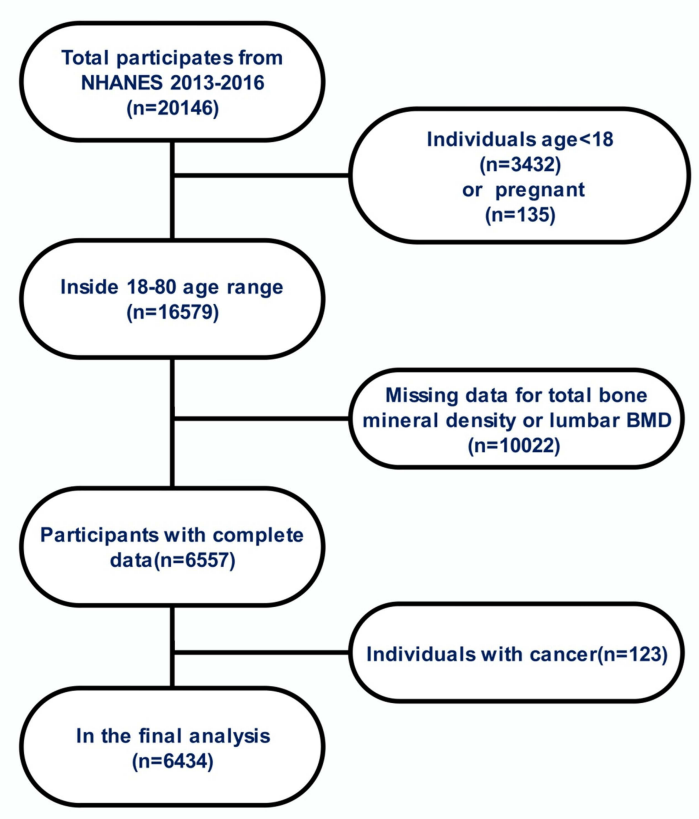

Figure I Research flow chart.

\section{The Relationship Between Variables Was Assessed Using the Pearson Coefficient}

Pearson correlation was calculated to assess the strength of relationship between fasting blood glucose, sex hormones and BMD among 6434 participates (Table 2). The fasting blood glucose $(\mathrm{FBG})$ and testosterone were correlated positively with thoracic spine BMD $(p<0.001, r=0.105$; $p<0.001, r=0.119)$. Estradiol was correlated positively with lumbar spine BMD and pelvis BMD $(p<0.001$, $r=0.115 ; p<0.001, r=0.099)$. SHBG showed a negative correlation with thoracic spine BMD and total BMD $(p<0.001, r=-0.117 ; p<0.001, r=-0.126)$ with positive correlation with lumbar spine BMD $(p<0.001, r=-0.049)$ according to Pearson relationship analysis.

\section{Associations Between Sex Steroid Hormone (nmol/L) and Lumbar BMD}

We selected confounders on the basis of their associations with the outcomes of interest or a change in effect estimate of more than $10 \%$. In the adjusted analysis (Table 3 ) the effects were adjusted for age, BMI, ALB, ALK, ASP, calcium, cholesterol, potassium, sodium, total protein, uric acid. No effect was found between FBG and lumbar BMD in two models. The results remained after adjusting for confounders (unadjusted: $\beta=0.02,95 \% \mathrm{CI}$ : 0.98-1.07; adjusted: $\beta=0.004$, 
Table I Weighted Characteristics of Study Sample Based on Age

\begin{tabular}{|c|c|c|c|c|}
\hline & $\begin{array}{l}\text { Age }<30 \text { Young } \\
(n=2137)\end{array}$ & $\begin{array}{l}\text { Age } 30-50 \text { Mid-Age } \\
(n=3033)\end{array}$ & $\begin{array}{l}\text { Age }>50 \text { Elderly } \\
(n=\mid 264)\end{array}$ & $p$-value \\
\hline \multicolumn{5}{|l|}{ Gender (n) } \\
\hline Male & 1094 & 1459 & 622 & \\
\hline Female & 1043 & 1574 & 642 & \\
\hline \multicolumn{5}{|l|}{ Education level (n) } \\
\hline Less than 9th grade & 52 & 247 & 121 & \\
\hline 9-I Ith grade & 189 & 369 & 159 & \\
\hline High school graduate/GED or equivalent & 374 & 628 & 304 & \\
\hline Some college or AA degree & 617 & 910 & 366 & \\
\hline College graduate or above & 394 & 879 & 314 & \\
\hline Body mass index $\left(\mathrm{kg} / \mathrm{m}^{2}\right)$ & $27.17 \pm 7.17$ & $29.60 \pm 6.92$ & $29.65 \pm 6.67$ & \\
\hline Fasting glucose (mmol/L) & $5.30 \pm 0.97$ & $5.91 \pm 2.02 *$ & $6.45 \pm 2.39 * * *$ & $<0.01$ \\
\hline $\mathrm{DBP}(\mathrm{mmHg})$ & $65.09 \pm 11.25$ & $72.82 \pm 10.97^{*}$ & $73.77 \pm 11.65^{*}, * *$ & $<0.01$ \\
\hline SBP $(\mathrm{mmHg})$ & $113.64 \pm 10.83$ & $119.20 \pm 14.39 *, * *$ & $126.72 \pm 17.95 * * *$ & $<0.01$ \\
\hline Total BMD $\left(\mathrm{g} / \mathrm{cm}^{2}\right)$ & $I . I I \pm 0.1 I$ & $1.12 \pm 0.11 *$ & $1.08 \pm 1.12 * * *$ & $<0.01$ \\
\hline Thoracic spine BMD $\left(\mathrm{g} / \mathrm{cm}^{2}\right)$ & $0.80 \pm 0.10$ & $0.83 \pm 0.1 I^{*}$ & $0.80 \pm 0.14 * *$ & $<0.01$ \\
\hline Lumbar spine BMD $\left(\mathrm{g} / \mathrm{cm}^{2}\right)$ & $1.04 \pm 0.14$ & $1.03 \pm 0.15$ & $0.99 \pm 0.16 * *$ & $<0.01$ \\
\hline Pelvis BMD $\left(\mathrm{g} / \mathrm{cm}^{2}\right)$ & $1.24 \pm 0.17$ & $1.26 \pm 0.16^{*}$ & I. $19 \pm 0.17 * * *$ & $<0.01$ \\
\hline Right leg BMD $\left(\mathrm{g} / \mathrm{cm}^{2}\right)$ & $1.18 \pm 0.14$ & $1.17 \pm 0.13$ & $1.14 \pm 0.14 * * *$ & $<0.01$ \\
\hline Right arm BMD $\left(\mathrm{g} / \mathrm{cm}^{2}\right)$ & $0.79 \pm 0.98$ & $0.80 \pm 0.10$ & $0.77 \pm 0.11 * * *$ & $<0.01$ \\
\hline Left leg BMD $\left(\mathrm{g} / \mathrm{cm}^{2}\right)$ & $1.17 \pm 0.14$ & $1.17 \pm 0.13$ & $1.13 \pm 0.14 * * *$ & $<0.01$ \\
\hline Left arm BMD $\left(\mathrm{g} / \mathrm{cm}^{2}\right)$ & $0.77 \pm 0.09$ & $0.78 \pm 0.10 *$ & $0.75 \pm 0.11 * * *$ & $<0.01$ \\
\hline \multicolumn{5}{|l|}{ Serum index } \\
\hline Albumin $(\mathrm{g} / \mathrm{dL})$ & $4.45 \pm 0.33$ & $4.31 \pm 0.32 *$ & $4.27 \pm 0.31 * * *$ & $<0.01$ \\
\hline ALk (IU/L) & $65.23 \pm 19.66$ & $65.07 \pm 22.28$ & $72.45 \pm 25.69 * * * *$ & $<0.01$ \\
\hline ASA (IU/L) & $25.07 \pm 23.36$ & $25.70 \pm 21.70$ & $26.83 \pm 15.94$ & \\
\hline ALA (IU/L) & $25.03 \pm 21.00$ & $27.05 \pm 20.74 *$ & $27.41 \pm 21.83 * * *$ & \\
\hline Bicarbonate $(\mathrm{mmol} / \mathrm{L})$ & $24.51 \pm 2.09$ & $24.60 \pm 2.14$ & $25.11 \pm 2.22 * * * *$ & $<0.01$ \\
\hline Total calcium (mg/dL) & $9.48 \pm 0.33$ & $9.32 \pm 0.36 *$ & $9.40 \pm 0.34 * * *$ & $<0.01$ \\
\hline Cholesterol (mg/dL) & $173.05 \pm 34.13$ & $196.53 \pm 40.61 *$ & $203.93 \pm 43.8 I^{*}, * *$ & $<0.01$ \\
\hline Globulin (g/dL) & $2.8 I \pm 0.39$ & $2.86 \pm 0.42^{*}$ & $2.84 \pm 0.45$ & $<0.01$ \\
\hline Potassium (mmol/L) & $3.94 \pm 0.30$ & $3.94 \pm 0.30$ & $3.98 \pm 0.36 *$ & $<0.01$ \\
\hline Sodium (mmol/L) & $139.21 \pm 1.98$ & $139.03 \pm 2.04 *$ & $139.37 \pm 2.36 * *$ & $<0.01$ \\
\hline Phosphorus (mg/dL) & $3.90 \pm 0.57$ & $3.7 I \pm 0.56 *$ & $3.79 \pm 0.58 * * *$ & $<0.01$ \\
\hline Total bilirubin $(\mathrm{mg} / \mathrm{dL})$ & $0.62 \pm 0.33$ & $0.59 \pm 0.29 *$ & $0.59 \pm 0.27 * * *$ & $<0.01$ \\
\hline Total protein $(\mathrm{g} / \mathrm{L})$ & $4 I .6 I \pm 32.5 I$ & $41.73 \pm 32.06$ & $39.44 \pm 31.83$ & \\
\hline Uric acid (umol/L) & $317.40 \pm 79.67$ & $313.33 \pm 82.92$ & $321.22 \pm 80.38$ & \\
\hline Testosterone (nmol/L) & $8.26 \pm 8.81$ & $6.74 \pm 7.95^{*}$ & $7.07 \pm 8.10^{*}$ & $<0.01$ \\
\hline Estradiol (nmol/L) & $0.19 \pm 0.28$ & $0.21 \pm 0.27$ & $0.08 \pm 0.13 *, * *$ & $<0.01$ \\
\hline SHBG (nmol/L) & $53.50 \pm 48.63$ & $54.78 \pm 38.38$ & $54.44 \pm 32.71$ & \\
\hline
\end{tabular}

Notes: Mean \pm SD for continuous variables; $p$-value was calculated by one-way ANOVA, $* p<0.01$ vs age $<30$ young; $* *<<0.01$ vs age $30-50$ mid-age.

Abbreviations: BMI, body mass index; SBP, systolic blood pressure; DBP, diastolic blood pressure; ALK, alkaline phosphatase; ASA, aspartate aminotransferase; ALA, alanine aminotransferase; BMD, bone mineral density.

95\%CI: 1.00-1.10). For lower lumbar BMD, all sex steroid hormone level were higher (testosterone: unadjusted $\beta=0.012$, 95\%CI: $1.00-1.02$; adjusted $\beta=0.020,95 \%$ CI: $1.01-1.03$; estradiol unadjusted $\beta=0.96,95 \% \mathrm{CI}: 1.82-3.75$; adjusted $\beta=0.76$, 95\%CI: $1.47-3.09$; SHBG unadjusted $\beta=-0.003$, 95\%CI: $1.00-1.01$; adjusted $\beta=0.10,95 \%$ CI: $1.00-1.01$ ). 
Table 2 Correlation Between BMD in Different Parts and Fasting Glucose/Sex Hormones

\begin{tabular}{|l|l|l|l|l|l|l|l|l|}
\hline \multirow{2}{*}{ Variables } & Fasting Blood Glucose & \multicolumn{2}{l|}{ Testosterone } & \multicolumn{2}{l|}{ Estradiol } & \multicolumn{2}{l|}{ SHBG } \\
\cline { 2 - 9 } & r-value & $p$-value & r-value & $p$-value & $r$-value & $p$-value & $r$-value & $p$-value \\
\hline Thoracic spine BMD $\left(\mathrm{g} / \mathrm{cm}^{2}\right)$ & 0.105 & $<0.001$ & 0.119 & $<0.001$ & 0.16 & 0.208 & -0.117 & $<0.001$ \\
Lumbar spine BMD $\left(\mathrm{g} / \mathrm{cm}^{2}\right)$ & 0.018 & 0.339 & 0.009 & 0.485 & 0.115 & $<0.001$ & 0.049 & $<0.001$ \\
Pelvis BMD $\left(\mathrm{g} / \mathrm{cm}^{2}\right)$ & 0.030 & 0.108 & -0.149 & 0.373 & 0.099 & $<0.001$ & 0.012 & 0.361 \\
Total BMD $\left(\mathrm{g}^{2} \mathrm{~cm}^{2}\right)$ & 0.033 & 0.080 & 0.223 & $<0.001$ & -0.020 & 0.107 & -0.126 & $<0.001$ \\
\hline
\end{tabular}

Notes: Pearson correlation was calculated to assess the strength of relationship between fasting blood glucose, sex hormones and BMD.

Table 3 Associations Between Sex Steroid Hormone (nmol/L) and Lumbar BMD

\begin{tabular}{|l|l|l|l|l|l|l|}
\hline \multirow{2}{*}{ Variables } & \multicolumn{2}{l}{ Unadjusted } & \multicolumn{3}{l|}{ Adjusted } \\
\cline { 2 - 7 } & $\boldsymbol{\beta}$ & OR (95\%Cl) & $\boldsymbol{p}$-value & $\boldsymbol{\beta}$ & OR (95\%Cl) & $\boldsymbol{p}$-value \\
\hline Fasting glucose & 0.02 & $1.02(0.98-1.07)$ & 0.32 & 0.04 & $1.05(1.00-1.10)$ & 0.051 \\
Testosterone & 0.012 & $1.01(1.00-1.02)$ & $<0.01$ & 0.02 & $1.02(1.01-1.03)$ & $<0.01$ \\
Estradiol & 0.96 & $2.61(1.82-3.75)$ & 0.013 & 0.76 & $2.13(1.47-3.09)$ & $<0.01$ \\
SHBG & 0.003 & $1.00(1.00-1.01)$ & $<0.01$ & 0.003 & $1.00(1.00-1.01)$ & $<0.01$ \\
\hline
\end{tabular}

Notes: Unadjusted: no covariates were adjusted; adjusted: age, BMI, ALB, ALK, ASP, calcium, cholesterol, potassium, sodium, total protein, uric acid. Effects are presented in both an unadjusted and an adjusted analysis with effect estimates and $p$-value. For this analysis 6434 participants were included.

\section{Associations Between Sex Steroid Hormone (nmol/L) and Pelvis BMD}

In the adjusted analysis (Table 4) the effects were adjusted for Age, BMI, ALB, ALK, ASP, calcium, cholesterol, potassium, sodium, total protein, uric acid. We found no effect between FBG and pelvis BMD in two models. The results remained after adjusting for confounders (unadjusted: $\beta=0.01$, OR $95 \%$ CI: $0.97-1.06$; adjusted: $\beta=0.01, \quad 95 \% \mathrm{CI}: \quad 0.96-1.05$ ). Testosterone and estradiol levels were higher with increased pelvis BMD (testosterone: unadjusted $\beta=0.007,95 \% \mathrm{CI}$ : 1.00-1.02; adjusted $\beta=0.014,95 \% \mathrm{CI}: 1.00-1.03$; estradiol: unadjusted $\beta=0.50,95 \% \mathrm{CI}: 1.18-2.33$; adjusted $\beta=0.42$, 95\% CI: 1.07-2.18). However, the opposite pattern was observed for SHBG relative change (unadjusted $\beta=-0.007$, 95\%CI: 0.99-1.00; adjusted $\beta=0.10,95 \%$ CI: $0.99-1.00$ ).

\section{Associations Between Sex Steroid Hormone (nmol/L) and Total BMD}

In the adjusted analysis (Table 5) the effects were adjusted for age, BMI, ALB, ALK, ASP, calcium, cholesterol, potassium, sodium, total protein, uric acid. There was no effect among FBG and testosterone to total BMD in two models $(p>0.05)$. For lower total BMD, estradiol level were higher and same effect was observed after adjusting for confounders (Estradiol Unadjusted $\beta=0.44$, 95\%CI: $1.12-2.13$; adjusted $\beta=0.39,95 \%$ CI: $1.05-2.07$ ). However, for lower total BMD, SHBG levels were higher with decreased total BMD and no significant effect was observed after adjusting for confounders (unadjusted $\beta=-0.002$, 95\%CI: $0.996-1.00$; adjusted $\beta=-0.001,95 \%$ CI: $1.00-1.002)$.

Table 4 Associations Between Sex Steroid Hormone (nmol/L) and Pelvis BMD

\begin{tabular}{|l|l|l|l|l|l|l|}
\hline \multirow{2}{*}{ Variables } & \multicolumn{2}{l}{ Unadjusted } & \multicolumn{3}{l|}{ Adjusted } \\
\cline { 2 - 7 } & $\boldsymbol{\beta}$ & OR (95\%Cl) & $\boldsymbol{p}$-value & $\boldsymbol{\beta}$ & OR (95\%Cl) & $\boldsymbol{p}$-value \\
\hline Fasting glucose & 0.01 & $1.01(0.97-1.06)$ & 0.662 & 0.01 & $1.00(0.96-1.05)$ & 0.84 \\
Testosterone & 0.007 & $1.01(1.00-1.02)$ & 0.116 & 0.014 & $1.01(1.00-1.03)$ & $<0.01$ \\
Estradiol & 0.50 & $1.66(1.18-2.33)$ & $<0.01$ & 0.42 & $1.52(1.07-2.18)$ & $<0.01$ \\
SHBG & -0.007 & $0.99(0.99-1.00)$ & $<0.01$ & 0.10 & $0.10(0.99-1.00)$ & $<0.01$ \\
\hline
\end{tabular}

Notes: Unadjusted: no covariates were adjusted; adjusted: age, BMI, ALB, ALK, ASP, calcium, cholesterol, potassium, sodium, total protein, uric acid. 
Table 5 Associations Between Sex Steroid Hormone (nmol/L) and Total BMD

\begin{tabular}{|l|l|l|l|l|l|l|}
\hline \multirow{2}{*}{ Variables } & \multicolumn{2}{l}{ Unadjusted } & \multicolumn{3}{l|}{ Adjusted } \\
\cline { 2 - 7 } & $\boldsymbol{\beta}$ & OR (95\%Cl) & $\boldsymbol{p}$-value & $\boldsymbol{\beta}$ & OR (95\%Cl) & $\boldsymbol{p}$-value \\
\hline Fasting glucose & 0.01 & $1.01(0.97-1.06)$ & 0.637 & -0.001 & $1.00(0.95-1.05)$ & 0.96 \\
Testosterone & 0.05 & $1.05(1.04-1.06)$ & 0.116 & 0.06 & $1.06(1.05-1.07)$ & $<0.01$ \\
Estradiol & 0.44 & $1.54(1.12-2.13)$ & $<0.01$ & 0.39 & $1.48(1.05-2.07)$ & 0.026 \\
SHBG & -0.002 & $0.998(0.996-1.00)$ & 0.02 & -0.001 & $1.00(1.00-1.002)$ & 0.56 \\
\hline
\end{tabular}

Notes: Unadjusted: no covariates were adjusted; adjusted: age, BMI, ALB, ALK, ASP, calcium, cholesterol, potassium, sodium, total protein, uric acid.

\section{Compared Sex Steroid Hormone (nmol/L) with Fasting Glucose to BMD}

According to the FBG and sex steroid hormone level, participants were divided into four groups. Group 1: FBG $\leq 6.0$ and low sex steroid hormone level; group 2: FBG $>6.0$ and low sex steroid hormone level; group 3: FBG $\leq 6.0$ and high sex steroid hormone level; group 4: FBG $>6.0$ and high sex steroid hormone level. Comparison of lumbar spine BMD, pelvis BMD and total BMD among four groups were shown in Table 6 and Figure 2. Our analysis showed that lumbar spine $\mathrm{BMD}$, pelvis BMD and total BMD were all significant increased in group 3 compared with group 1 and group 2. The results indicated that high level of sex steroid hormone level increased BMD, whereas FBG had no significant effect on BMD.

\section{Explore Differences in the Recording of Indicator Index Between Genders}

Next, participants were grouped into males $(n=3175)$ and females $(n=3259)$ as shown in Table 8 . The mean age in males was $37.39 \pm 12.40$ compared to group of females with a mean age of $37.95 \pm 12.13$ ( $p=0.087$ ). Males had a lower BMI of $28.26 \pm 6.25$ compared to $29.32 \pm 7.72$ for females. However, notably, the fasting glucose was higher for males $(5.95 \pm 1.92$ vs $5.71 \pm 1.86)$. Also, BMD indexes were lower in females, whereas SHBG were higher in females $(54.78 \pm 38.38)$ compared to males $(53.50 \pm 48.63)$.

\section{Multivariate Regression Analysis of SHBG on BMD in Different Genders}

We investigated data to explore whether SHBG are significant predictors of BMD by multivariate regression analysis in different genders (Table 7). Among all the subjects, serum SHBG level was statistically significant among the subgroups. Compared with females, males with higher level of SHBG had significantly decreased in thoracic spine BMD (OR: 0.271; 95\%CI, 0.073-0.469; $p=0.007$ ), lumbar spine BMD (OR: 0.572; 95\%CI, 0.292-0.852; $p<0.001$ ), pelvis BMD (OR: $0.681 ; 95 \% \mathrm{CI}, 0.381-0.982 ; p<0.0001)$ and total BMD (OR: 0.722; 95\%CI, 0.520-0.9250; $p<0.001$ ) in model 3. Our data also revealed that the level of SHBG was protective factor in lumbar spine BMD (OR: 1.044; 95\%CI,1.011-1.077; $p<0.001)$, pelvis BMD (OR: $1.1092 ; 95 \% \mathrm{CI}, 1.164-1.220 ; p<0.001$ ), total BMD (OR: $1.017 ; 95 \% \mathrm{CI}, 0.979-1.055 ; p<0.001$ ) in females. Combined with Table 8, the level of SHBG was higher in females than males. Our results demonstrated that SHBG levels were significant associated with BMD in the multivariate model (adjusted for: age, BMI, PIR, fasting blood glucose, calcium, cholesterol, total protein, uric acid, testosterone and estradiol), however, it will be a different effect between the genders. Sex hormones influence bone acquisition and metabolism. Men develop wider bones and greater cortical bone size than women due to periosteal apposition.

Table 6 Compared Sex Steroid Hormone (nmol/L) with Fasting Glucose to BMD

\begin{tabular}{|l|l|l|l|l|}
\hline & Group I $(\mathbf{n}=100)$ & Group 2 $(\mathbf{n = 4 6 )}$ & Group 3 $(\mathbf{n = 1 6 9 )}$ & Group 4 (n=43) \\
\hline Lumbar spine BMD $\left(\mathrm{g} / \mathrm{cm}^{2}\right)$ & $0.891 \pm 0.164$ & $0.952 \pm 0.133$ & $1.048 \pm 0.153^{*}, * *$ & $1.045 \pm 0.150^{*}, * *$ \\
Pelvis BMD $\left(\mathrm{g} / \mathrm{cm}^{2}\right)$ & $1.189 \pm 0.160$ & $1.150 \pm 0.162$ & $1.251 \pm 0.175^{*}, * *$ & $1.250 \pm 0.153^{* *}$ \\
Total BMD $\left(\mathrm{g} / \mathrm{cm}^{2}\right)$ & $1.050 \pm 0.114$ & $1.043 \pm 0.113$ & $1.149 \pm 0.104 *, * *$ & $1.151 \pm 0.092^{*}, * *$ \\
\hline
\end{tabular}

Notes: ${ }^{*} p<0.05$ vs group I; ${ }^{* *} p<0.05$ vs group 2 . 


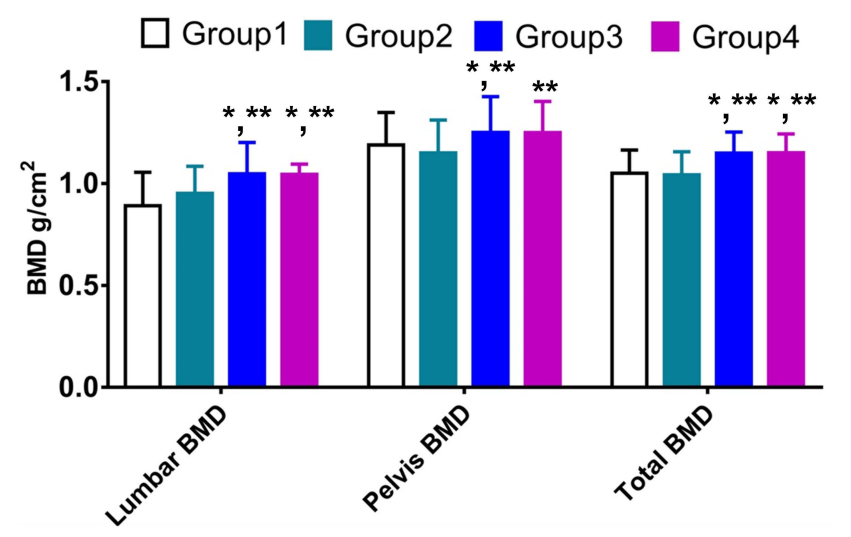

Figure 2 Group I: FBG $\leq 6.0$ and low sex steroid hormone level; group 2: FBG $>6.0$ and low sex steroid hormone level; group 3 : FBG $\leq 6.0$ and high sex steroid hormone level; group 4: FBG $>6.0$ and high sex steroid hormone level. The data are presented as the means \pm SD. Statistical analysis was performed by two-way analysis of variance with all pairwise multiple comparison procedures done by Tukey's method, $* p<0.001$ vs group I; $* * p<0.001$ vs group 2 .

\section{Discussion}

Osteoporosis is a systemic bone disorder with degradation of skeletal micro-architecture, which can impact a variety of bones throughout the body. BMD is affected by age, gender, sex hormone level, smoke, and lifestyle. ${ }^{16}$ Currently, due to lack of awareness as well as techniques in osteoporosis, it is difficult to achieve the processes of standardized diagnosis and treatment for clinicians. Personal and societal costs of osteoporosis are increasing as the ageing population increases. Aside from age, gender and diabetes status are considered to attribute to osteoporosis, ${ }^{17}$ which represents a silent epidemic that carries significant mortality and morbidity. The main focus of clinicians is to detect the factor which may effect osteoporosis as early as possible.

We collected 6343 participants in NHANES 2013-2016 and our analysis showed that FBG was increased with age from 30 to 80 years. But the evidence of such a causal relationship between FBG and BMD is not completely consistent ( $p>0.05$, Table 2). FBG did not appear to be associated with lumbar BMD, pelvic BMD and total BMD whether we have adjusted for confounding factors ( $p>0.05$, Tables $3-5)$, which is not consistent with previous studies. ${ }^{18-20}$

Circulating SHBG levels are inversely associated with the risk of developing type 2 diabetes and insulin resistance. Previous studies summarized that bone abnormalities caused by diabetes are all based on those whose blood glucose itself is not well controlled, leading to unhealthy physiological and biochemical changes. The BMD or other indexes could be better than those whose FBG is not wellcontrolled, even if the FBG is a little higher (no oral antiglycemic agents or insulin). This means that the underlying mechanism and influencing factors in formation of osteoporosis are complex in our studies.

It has been shown that BMD often decreases substantially after the age of 50, and BMD has been associated with variations in sex hormones. ${ }^{21,22}$ It is known that sex hormone levels or functional hormone receptors will decrease with age, which plays a significant role in the osteoporosis process. ${ }^{11}$ Estrogens exert their regulatory potential on exhibiting the upregulating effect through subunit estrogen receptor (ER). ER- $\alpha$ is one part of PGC- $1 \alpha$, which is regarded as the key regulator of energy homeostasis and mitochondrial biogenesis. ${ }^{23}$ Estrogen prevents bone loss via ER- $\alpha$ to induce downstream genomic signaling and induction of Fas ligand in osteoclasts. ${ }^{24}$ Selective estrogen receptor modulators (SERMs) have been regarded as a double role in slowing down the bone loss and inhibiting joint inflammation, but, only using estrogen has no protective effect on chondrocytes in

Table 7 Multivariate Regression Analysis of SHBG on BMD

\begin{tabular}{|c|c|c|c|c|}
\hline Variables & Sex & Model I OR (95\%Cl) p-value & Model 2 OR, (95\%Cl), p-value & Model 3 OR $(95 \% \mathrm{Cl})$ p-value \\
\hline \multirow[t]{2}{*}{ Thoracic spine BMD $\left(\mathrm{g} / \mathrm{cm}^{2}\right)$} & Male & $0.834(0.819-0.848)<0.001$ & $0.598(0.561-0.635)<0.001$ & $0.27 \mid(0.073-0.469) 0.007$ \\
\hline & Female & $0.82(0.795-0.808)<0.0001$ & $0.675(0.656-0.695)<0.001$ & $0.676(0.65 I-0.700)<0.00 I$ \\
\hline \multirow[t]{2}{*}{ Lumbar spine BMD $\left(\mathrm{g} / \mathrm{cm}^{2}\right)$} & Male & $1.014(0.995-1.032)<0.001$ & $0.949(0.899-0.999)<0.001$ & $0.572(0.292-0.852)<0.001$ \\
\hline & Female & $1.022(1.014-1.031)<0.001$ & $1.028(1.002-1.055)<0.001$ & $1.044(1.011-1.077)<0.001$ \\
\hline \multirow[t]{2}{*}{ Pelvis BMD $\left(\mathrm{g} / \mathrm{cm}^{2}\right)$} & Male & $1.311(1.290-1.332)<0.001$ & $1.127(1.072-1.18 I)<0.00 I$ & $0.68 \mathrm{I}(0.38 \mathrm{I}-0.982)<0.000 \mathrm{I}$ \\
\hline & Female & $1.230(1.221-1.239)<0.001$ & $1.209(1.182-1.235)<0.001$ & $1.1092(1.164-1.220)<0.0001$ \\
\hline \multirow[t]{2}{*}{ Total BMD $\left(\mathrm{g} / \mathrm{cm}^{2}\right)$} & Male & $1.141(1.128-1.155)<0.001$ & $1.054(1.017-1.09)<0.001$ & $0.722(0.520-0.925)<0.001$ \\
\hline & Female & $1.076(1.067-1.086)<0.001$ & $1.01(0.979-1.049)<0.001$ & $1.017(0.979-1.055)<0.001$ \\
\hline
\end{tabular}

Notes: Model I is adjusted for no variables; model 2 is adjusted for age, BMI, PIR, fasting blood glucose; model 3 is adjusted for calcium, cholesterol, total protein, uric acid, testosterone and estradiol. 
Table 8 Compared Sex Steroid Hormone (nmol/L) with Sex to BMD

\begin{tabular}{|l|l|l|l|}
\hline & Male $(\mathbf{n}=\mathbf{3}$ I 75) & Female $(\mathbf{n = 3 2 5 9 )}$ & p-value \\
\hline Age & $37.39 \pm 12.40$ & $37.95 \pm 12.13$ & 0.087 \\
\hline Body mass index $\left(\mathrm{kg} / \mathrm{m}^{2}\right)$ & $28.26 \pm 6.25$ & $29.32 \pm 7.72^{*}$ & $<0.01$ \\
\hline Fasting glucose $(\mathrm{mmol} / \mathrm{L})$ & $5.95 \pm 1.92$ & $5.71 \pm 1.86$ & 0.414 \\
\hline Total BMD $\left(\mathrm{g} / \mathrm{cm}^{2}\right)$ & $1.14 \pm 0.11$ & $1.08 \pm 0.10^{*}$ & $<0.01$ \\
Thoracic spine BMD $\left(\mathrm{g} / \mathrm{cm}^{2}\right)$ & $0.84 \pm 0.12$ & $0.79 \pm 0.11$ & 0.067 \\
Lumbar spine BMD $\left(\mathrm{g} / \mathrm{cm}^{2}\right)$ & $1.03 \pm 0.16$ & $1.03 \pm 0.14^{*}$ & 0.01 \\
Pelvis BMD $\left(\mathrm{g} / \mathrm{cm}^{2}\right)$ & $1.27 \pm 0.17$ & $1.21 \pm 0.15^{*}$ & $<0.01$ \\
Right leg BMD $\left(\mathrm{g} / \mathrm{cm}^{2}\right)$ & $1.24 \pm 0.13$ & $1.10 \pm 0.11^{*}$ & $<0.01$ \\
Right arm BMD $\left(\mathrm{g} / \mathrm{cm}^{2}\right)$ & $0.85 \pm 0.08$ & $0.72 \pm 0.07^{*}$ & $<0.01$ \\
Left leg BMD $\left(\mathrm{g} / \mathrm{cm}^{2}\right)$ & $1.23 \pm 0.13$ & $0.70 \pm 0.17^{*}$ & $<0.01$ \\
Left arm BMD $\left(\mathrm{g} / \mathrm{cm}^{2}\right)$ & $0.83 \pm 0.08$ & $6.74 \pm 7.95^{*}$ & $<0.01$ \\
\hline Testosterone $(\mathrm{nmol} / \mathrm{L})$ & $8.26 \pm 8.81$ & $0.21 \pm 0.27^{*}$ & $<0.01$ \\
Estradiol $(\mathrm{nmol} / \mathrm{L})$ & $0.19 \pm 0.28$ & $54.78 \pm 38.38^{*}$ & $<0.01$ \\
SHBG $(\mathrm{nmol} / \mathrm{L})$ & $53.50 \pm 48.63$ & $<0.01$ \\
\hline
\end{tabular}

Notes: Data are presented as the means \pm SEM. One-way ANOVA followed by a Holm-Sidak test for post-hoc analysis using GraphPad Prism 7 . A value of $p<0.05$ was considered to indicate a statistically significant difference. ${ }^{*} p$ vs male.

animal model. ${ }^{25}$ One of the strongest regulators of SHBG is hepatocyte nuclear factor 4 alpha (HNF 4- $\alpha$ ). HNF 4- $\alpha$ regulates the transcription of multiple target genes implicated in glucose metabolism and insulin secretion. ${ }^{26}$ It was not clear that whether SHBG was an independent predictor of osteoporosis. Multiple hormonal adaptations, designed to decrease energy expenditure during periods of low energy intake may be to blame for osteoporosis. In a threeyear research on male osteoporosis, SHBG positively correlated with bone absorption markers (C-telopeptide of type I collagen and free deoxypyridinoline) after adjustment for age, BMI, and sex steroids both in health controls and postmenopausal osteoporosis patients. ${ }^{11}$

Our data showed that total BMD, thoracic spine BMD, pelvis BMD and left arm BMD were increased in the midage group compared with other groups. Notably, total calcium in mid-age group was lower than other groups. The difference may be caused by increased bone turnover, bone formation, and decreased bone reabsorption. Another possibility is that calcitonin disorders can lead to abnormal bone metabolism. Calcitonin is one of the antiosteoporosis drugs, which may influence the regeneration of subchondral bone with the direct beneficial effects on articular cartilage by binding with calcitonin receptor on osteoclast and inhibiting bone resorption. ${ }^{27}$ There was no difference between young and mid-aged groups in arm $\mathrm{BMD}$ or leg BMD, but compared with the young group,
BMD was significantly decreased in the elderly group. Our data showed that no significant linear relationship was detected along with BMD among the age groups, but form an inverted U-shape throughout the age range. Pearson correlation analysis indicated that total BMD and thoracic spine BMD have significant positive correlation with testosterone (Table 2), lumbar BMD and pelvis BMD have significant positive correlation with estradiol. However, thoracic spine BMD and total BMD have significant negative correlation with SHBG at $p<0.05$ level whereas, SHBG has positive correlation with lumbar BMD. The effect of sex hormone on bone mineral density is relatively slow, while it has inconsistent effect on the BMD in different parts (Table 3). One of the reasons may be that increased high physical and social activity along with the increased BMD. ${ }^{28}$ The pelvis BMD will be increased, because the pelvis is a major structure which in conjunction with upper and lower body and withstands the weight of a human body. ${ }^{29,30}$ On the contrary, little effect of traction or external rotation on thoracic spine and the main effect of sex steroid hormones was not statistically significant with total BMD. Meanwhile, the experimental results are not consistent with theoretical results based on the different baseline of BMD, and the ratio of bone loss will not be exactly identical. Our results demonstrated that high level of sex steroid hormone levels increased BMD, whereas FBG had no significant 
effect on BMD. SHBG levels were significantly associated with BMD in the multivariate model, however, it will be a different effect between the genders.

\section{Limitations}

First, our study was a cross-sectional study to explore the relationship between sex hormones, blood glucose and BMD by dual energy X-ray. We indirectly speculated that factors may affect osteoporosis, while the role of sex hormones at genetically or protein levels of human should be demonstrated by prospective studies and experiments.

Second, it may have lacked statistical power or some major strengths, such as lifestyle habits, exercise, smoking, cancer, cardiovascular risk score were not adjusted.

Third, the sample size was reduced due to incomplete data collection on BMD of pelvis, lumbar and thoracic spine by dual energy X-ray in the NHANES database.

Finally, the findings are mainly applicable to the US population and cannot be extrapolated to other countries. Further longitudinal studies are needed to replicate the influence of osteoporosis on the association between sex hormones and SHBG.

\section{Abbreviations}

BMD, bone mineral density; AGEs, advanced glycation end products; SHBG, sex hormone-binding globulin; NHANES, National Health and Nutrition Examination Survey; NCHS, National Center for Health Statistics; FBG, fasting blood glucose.

\section{Availability of Supporting Data}

The authors declare that the materials described in the manuscript, including all relevant raw data, will be freely available to any scientist wishing to use them for noncommercial purposes, without breaching participant confidentiality. The ethics review board of the National Center for Health Statistics approved all NHANES protocols and written informed consents were obtained from all participants. Data was extracted from the 2013-2016 National Health and Nutrition Examination Survey (NHANES, n=6343), which was designed and conducted by the National Center for Health Statistics (NCHS) and freely available on the Internet for researchers throughout the world. The survey protocol was approved by the Institutional Review Board of the Centers for Disease Control and Prevention (protocol \#98-12 and protocol \#2005-2006). Written informed consent was obtained from all participants.

\section{Ethics Approval and Consent to Participate}

The ethics review board of the National Center for Health Statistics approved all NHANES protocols and written informed consents were obtained from all participants.

\section{Acknowledgments}

The authors thank the staff and the participants who contributed to the NHANES data, including all of the anonymous participants in the study. We also thank $\mathrm{Dr} \mathrm{Bo} \mathrm{Yu}$ from Key Laboratory of Myocardial Ischemia, Ministry of Education, Harbin Medical University (Harbin, China) who was more than generous with his expertise and precious time. His vigorous academic observation enlightens us not only in this thesis, but also in our future study.

\section{Author Contributions}

All authors made a significant contribution to the work reported, whether that is in the conception, study design, acquisition of data, analysis and interpretation, or in all these areas; took part in drafting, revising or critically reviewing the article; gave final approval of the version to be published; have agreed on the journal to which the article has been submitted; and agree to be accountable for all aspects of the work.

\section{Funding}

The present study was supported by grants from National Natural Science Foundation of China (no. 81901853) to FY. This study was also supported by Fund of Key Laboratory of Myocardial Ischemia, Ministry of Education (KF202010) and Innovation Fund of Harbin Medical University to JW. The funders had no conflict in the study design, data collection and analysis, decision to publish, or preparation of the manuscript.

\section{Disclosure}

The authors report no conflicts of interests in this work.

\section{References}

1. Wu D, Wu XD, Zhou X, Huang W, Luo C, Liu Y. Bone mineral density, osteopenia, osteoporosis, and fracture risk in patients with atopic dermatitis: a systematic review and meta-analysis. Ann Transl Med. 2021;9(1):40. doi:10.21037/atm-20-4708

2. Rinonapoli G, Ruggiero C, Meccariello L, Bisaccia M, Ceccarini P, Caraffa A. Osteoporosis in men: a review of an underestimated bone condition. Int J Mol Sci. 2021;22(4):2105. doi:10.3390/ijms22042105 
3. Almohaileb FI, Rasheed Z. Comparing the efficacies of bisphosphonates' therapies for osteoporosis persistence and compliance: a systematic review. Curr Mol Med. 2021;21. doi:10.2174/ 1566524021666210414100227

4. Elonheimo H, Lange R, Tolonen H, Kolossa-Gehring M. Environmental substances associated with osteoporosis-a scoping review. Int $J$ Environ Res Public Health. 2021;18(2):738. doi:10.3390/ijerph18020738

5. Lee A, Hassannia F, Bergin MJ, Al Zaabi K, Misale P, Rutka JA. The relationship between disorders of bone metabolism and benign paroxysmal positional vertigo: a systematic review. Ear Hear. 2021. doi:10.1097/AUD.0000000000001063

6. Rondanelli M, Faliva MA, Infantino V, et al. Copper as dietary supplement for bone metabolism: a review. Nutrients. 2021;13 (7):2246. doi:10.3390/nu13072246

7. Zhang R, Mao J, Wang X, et al. Increased bone mineral density in male patients with idiopathic hypogonadotropic hypogonadism who undergo sex hormone therapy: findings from cross-sectional and longitudinal studies. Endocr Pract. 2021;27(9):934-940. doi:10.1016/j.eprac.2021.05.004

8. Qu Z, Jiang J, Yang F, Huang J, Zhao J, Yan S. Genetically predicted sex hormone-binding globulin and bone mineral density: a Mendelian Randomization Study. Calcif Tissue Int. 2021;108(3):281-287. doi:10.1007/s00223-020-00770-8

9. Veldhuis-Vlug AG, Woods GN, Sigurdsson S, et al. Serum FSH is associated with BMD, bone marrow adiposity, and body composition in the AGES-Reykjavik Study of older adults. J Clin Endocrinol Metab. 2021;106(3):e1156-e1169. doi:10.1210/clinem/dgaa922

10. Champakanath A, Keshawarz A, Pyle L, Snell-Bergeon JK, Shah VN. Fracture risk assessment (FRAX) without BMD and risk of major osteoporotic fractures in adults with type 1 diabetes. Bone. 2021;143:115614. doi:10.1016/j.bone.2020.115614

11. Paller CJ, Shiels MS, Rohrmann S, et al. Relationship of sex steroid hormones with bone mineral density (BMD) in a nationally representative sample of men. Clin Endocrinol (Oxf). 2009;70(1):26-34. doi:10.1111/j.1365-2265.2008.03300.x

12. Zeng H, Yang Z, Li J, et al. Associations between female lung cancer risk and sex steroid hormones: a systematic review and meta-analysis of the worldwide epidemiological evidence on endogenous and exogenous sex steroid hormones. BMC Cancer. 2021;21(1):690. doi:10.1186/s12885-021-08437-9

13. Emami MR, Safabakhsh M, Khorshidi M, et al. Effect of bariatric surgery on endogenous sex hormones and sex hormone-binding globulin levels: a systematic review and meta-analysis. Surg Obes Relat Dis. 2021;17(9):1621-1636. doi:10.1016/j.soard.2021.05.003

14. Jing Y, Wang X, Yu J, et al. Associations of serum sex hormone binding globulin with bone mineral densities and higher 10-year probability of fractures in postmenopausal women with type 2 diabetes mellitus. Ann Transl Med. 2019;7(18):457. doi:10.21037/ atm.2019.08.46

15. Eriksson AL, Lorentzon M, Mellström D, et al. SHBG gene promoter polymorphisms in men are associated with serum sex hormone-binding globulin, androgen and androgen metabolite levels, and hip bone mineral density. J Clin Endocrinol Metab. 2006;91 (12):5029-5037. doi:10.1210/jc.2006-0679

16. Alonso Perez JL, Martin Perez S, Battaglino A, Villafane JH, AlonsoSal A, Sanchez Romero EA. An update of the muscle strengthening exercise effectiveness in postmenopausal women with osteoporosis: a qualitative systematic review. J Clin Med. 2021;10(11):2229. doi: $10.3390 /$ jcm 10112229
17. Migliorini F, Maffulli N, Spiezia F, Tingart M, Maria PG, Riccardo G. Biomarkers as therapy monitoring for postmenopausal osteoporosis: a systematic review. J Orthop Surg Res. 2021;16 (1):318. doi:10.1186/s13018-021-02474-7

18. Ebrahimpur M, Sharifi F, Nezhad FA, et al. Effect of diabetes on BMD and TBS values as determinants of bone health in the elderly: Bushehr elderly health program. J Diabetes Metab Disord. 2019;18 (1):99-106. doi:10.1007/s40200-019-00395-1

19. Danielson KK, Elliott ME, LeCaire T, Binkley N, Palta M. Poor glycemic control is associated with low BMD detected in premenopausal women with type 1 diabetes. Osteoporos Int. 2009;20 (6):923-933. doi:10.1007/s00198-008-0763-3

20. Komatsu Y, Majima T. Negative correlation between BMD and HbA1C in patients with type 2 diabetes. Clin Calcium. 2006;16 (8):1327-1331.

21. Zhang X, Hua T, Zhu J, et al. Body compositions differently contribute to BMD in different age and gender: a pilot study by QCT. Arch Osteoporos. 2019;14(1):31. doi:10.1007/s11657-019-0574-5

22. Papaioannou A, Kennedy CC, Cranney A, et al. Risk factors for low BMD in healthy men age 50 years or older: a systematic review. Osteoporos Int. 2009;20(4):507-518. doi:10.1007/s00198-008-07 20-1

23. Mirebeau-Prunier D, Le Pennec S, Jacques C, et al. Estrogen-related receptor alpha and PGC-1-related coactivator constitute a novel complex mediating the biogenesis of functional mitochondria. FEBS J. 2010;277(3):713-725. doi:10.1111/j.1742-4658.2009.07516.x

24. Nakamura T, Imai Y, Matsumoto T, et al. Estrogen prevents bone loss via estrogen receptor alpha and induction of fas ligand in osteoclasts. Cell. 2007;130(5):811-823. doi:10.1016/j.cell.2007.07.025

25. Sato Y, Tando T, Morita M, et al. Selective estrogen receptor modulators and the vitamin D analogue eldecalcitol block bone loss in male osteoporosis. Biochem Biophys Res Commun. 2017;482 (4):1430-1436. doi:10.1016/j.bbrc.2016.12.053

26. Li CX, Tang ST, Zhang Q. Changes in bone mineral density and metabolic parameters after pulsatile gonadorelin treatment in young men with hypogonadotropic hypogonadism. Int $J$ Endocrinol. 2015;2015:324524. doi:10.1155/2015/324524

27. Paniccia R, Riccioni T, Zani BM, Zigrino P, Scotlandi K, Teti A. Calcitonin down-regulates immediate cell signals induced in human osteoclast-like cells by the bone sialoprotein-IIA fragment through a postintegrin receptor mechanism. Endocrinology. 1995;136 (3):1177-1186. doi:10.1210/endo.136.3.7867571

28. Alkahtani S, Aljaloud K, Yakout S, Al-Daghri NM. Interactions between sedentary and physical activity patterns, lean mass, and bone density in Arab men. Dis Markers. 2019;2019:5917573. doi:10.1155/2019/5917573

29. Shon WY, Sharma V, Keon OJ, Moon JG, Suh DH. Can pelvic tilting be ignored in total hip arthroplasty? Int J Surg Case Rep. 2014;5 (9):633-636. doi:10.1016/j.ijscr.2014.07.015

30. Diniz ALL, Rodrigues NCP, Sampaio FJB, Favorito LA. Study of the renal parenchymal volume during the human fetal period. Int Braz J Urol. 2019;45(1):150-160. doi:10.1590/s1677-5538.ibju.2018.0538 


\section{Publish your work in this journal}

The International Journal of General Medicine is an international, peer-reviewed open-access journal that focuses on general and internal medicine, pathogenesis, epidemiology, diagnosis, monitoring and treatment protocols. The journal is characterized by the rapid reporting of reviews, original research and clinical studie across all disease areas. The manuscript management system is completely online and includes a very quick and fair peer-review system, which is all easy to use. Visit http://www.dovepress.com/ testimonials.php to read real quotes from published authors.

Submit your manuscript here: https://www.dovepress.com/international-journal-of-general-medicine-journal 